\title{
Notes sur le calcul hydraulique des grilles «par-dessous »
}

\section{Notes on the hydraulic design of bottom-type intake screens}

\author{
PAR GÉdÉon DAGAN (DRIMMER), \\ INGÉNIEUR AU LABORATOIRE D'HYDRAULIQUE DU TECHNION, \\ INSTITUT DE TEGHNOLOGIE D'ISRAËL
}

\begin{abstract}
L'étude théorique du mouvement permanent sur une grille "par-dessous 》 aboutit à l'établissement d'un système différentiel de quatre équations $\dot{a}$ cinq fonctions inconnues. A l'aide de ce système, on analyse qualitativement la forme de la nappe. Pour obtenir des résultats quantitatifs plus exacts, on ajoute une relation empirique basée sur l'étude expérimentale de M. Noseda (1956). On démontre qu'on peut étudier la distribution du débit le long de la grille, sans tenir compte de la forme de la nappe et une relation empirique adimensionnelle est établie dans ce but. A l'aide de cette relation, on peut déterminer la forme de la surface libre et la longueur de la grille. La méthode est illustrée par deux exemples de calcul.
\end{abstract}

\begin{abstract}
A theoretical study of the steady flow over a bottom-type intake screen leads to a differential system of four equations with five unknown functions, which is used for a qualitative analysis of the shape of the nappe. An empirical relation based on an experimental study by $M$. Noseda (1956) is then added in order to obtain more exact quantitative results. It is shown that the distribution of the flow rates along the screen can be studied without allowing for the shape of the nappe; a dimensionless empirical relation is established for the purpose, by means of which both the shape of the free surface and the length of the screen can be determined. Two calculated examples are given to illustrate the method.
\end{abstract}

1. Le problème hydraulique des grilles «pardessous 》 a été étudié par plusieurs auteurs [1], $[11],[14],[3],[9],[10],[8]$; usant de différentes hypothèses, on a déduit diverses formules.

Les expériences systématiques effectuées par M. Noseda [12] [13] ont démontré que la détermination du profil de la nappe et du débit dérivé par la grille à l'aide des équations :

- de l'orifice :

$$
d Q=\mu s \sqrt{2 g \mathrm{H}} d x
$$

- de l'énergie :

$$
\frac{d \mathrm{E}}{d x}=\frac{d}{d x}\left(\mathrm{H}+\frac{Q^{2}}{2 g \mathrm{H}^{2}}\right)=0
$$

- de continuité :

$$
d \mathrm{Q}=d(\mathrm{HU})
$$

n'aboutissent pas à des résultats satisfaisants. Pour obtenir une concordance entre les formules (1) et Jes expériences, M. Noseda a supposé que le coefficient de débit miné à l'aide des expériences.

Du point de vue de la théorie classique de l'orifice, ce résultat est paradoxal, parce que $\mu$ (coefficient de contraction) est plutôt une caractéristique géométrique de l'orifice et même si on superpose un mouvement de translation horizontal à celui vertical, $\mu$ reste constant.

D'autre part, les observations sur l'écoulement sur une grille à barreaux rares, emplacée sur la crête d'un barrage [16] ont démontré que la nappe peut être convexe; une telle forme ne résulte d'aucune méthode de calcul existante.

Dans les chapitres suivants, on aborde la question à la lumière de ces remarques. 

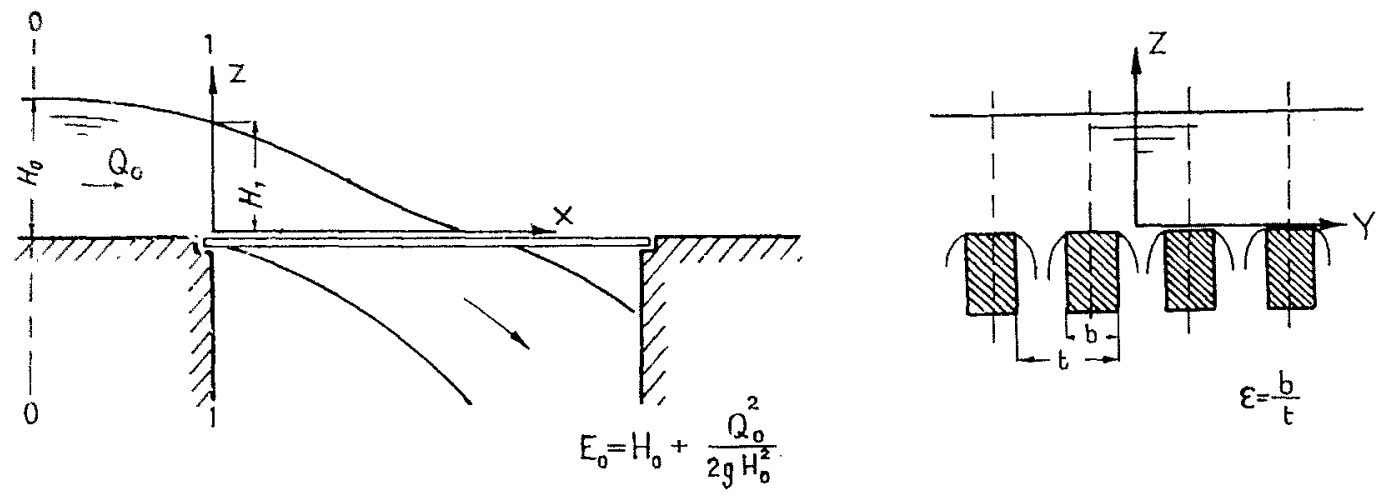

FIG. 1

\section{Hypothèses; équations fondamentales.}

On admet généralement, et les expériences ont démontré, que : (a) les pertes de charge au long de la grille sont négligeables [17] et le mouvement peut être considéré comme à potentiel; (b) la surface libre supérieure est cylindrique et l'on va supposer (c) que la configuration géométrique de la surface libre inférieure (à l'issue de Ia grille) est identique à celle d'un orifice (ou fente) sous pression « hydrostatique», c'est-à-dire que le coefficient de contraction et la distribution locale des vitesses sont similaires à ceux de ce dernier cas.

Usant de ces hypothèses, isolant par deux plans de symétrie une fente horizontale (avec les notations de la fig. 2), on peut écrire les équations suivantes :

$$
\frac{\mathrm{U}_{\mathrm{I}}^{2}(\mathrm{X})}{2 g}+\frac{\mathrm{W}_{\mathrm{I}}^{2}(\mathrm{X})}{2 g}+\mathrm{H}(\mathrm{X})=\mathrm{E}_{0} \quad(2 \alpha)
$$

(éq. de l'énergie à la surface libre).

$$
\frac{\mathrm{U}_{\mathrm{II}}^{2}(\mathrm{X})}{2 g}+\frac{\mathrm{V}_{\mathrm{II}}^{2}(\mathrm{X})}{2 g}=\mathrm{E}_{0}
$$

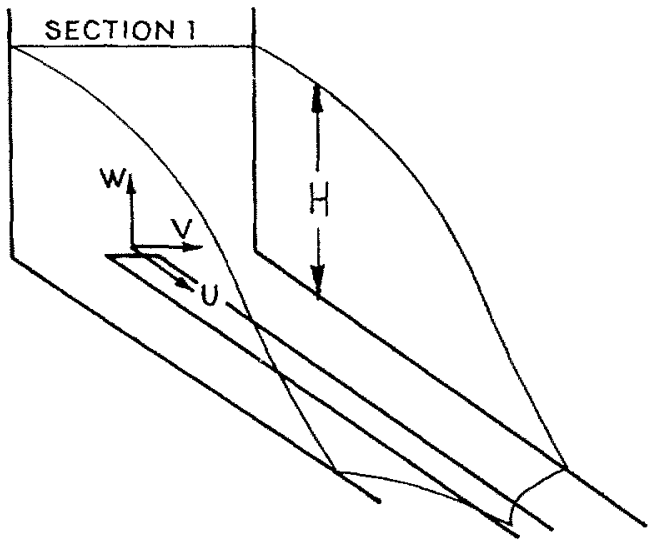

(éq. de l'énergie en $A$, fig. 2) ou :

$$
\begin{gathered}
\frac{\mathrm{U}_{\mathrm{II}}^{2}(\mathrm{X})}{2 g}+\mathrm{v}^{2} \frac{\mathrm{W}_{\mathrm{II}^{2}}(\mathrm{X})}{2 g}=\mathrm{E}_{0} \\
\frac{d \mathrm{H}(\mathrm{X})}{d \mathrm{X}}=\frac{\mathrm{W}_{\mathrm{I}}(\mathrm{X})}{\mathrm{U}_{\mathrm{I}}(\mathrm{X})}
\end{gathered}
$$

(éq. cinématique de la surface libre).

$$
d Q_{\text {derive }}=d Q_{\text {longit. }}
$$

(éc. de continuité)

Cette dernière équation peut être transformée ainsi :

$$
d \mathrm{Q}_{\text {dêrivé }}=\xi b \mathrm{~W}_{\mathrm{II}}(\mathrm{X}) d \mathrm{X}
$$

ou :

$$
\xi=\frac{\int_{0}^{b} \mathrm{~W}(\mathrm{X}, \mathrm{Y}, 0) d \mathrm{Y}}{b \mathrm{~W}_{\mathrm{II}}(\mathrm{X})}
$$

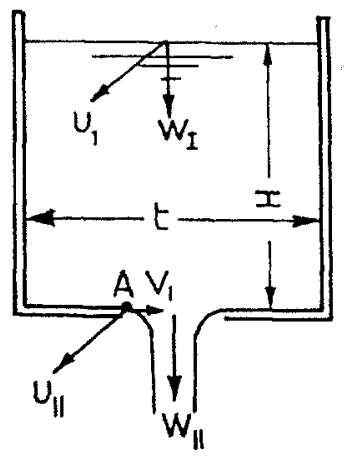

FIG. 2 
et :

$d \mathrm{Q}_{\text {longit. }}=\int_{0}^{t} d \mathrm{Y}\left[\int_{0}^{\mathrm{H}+d \mathrm{H}} \mathrm{U}(\mathrm{X}+d \mathrm{X}, \mathrm{Y}, \mathrm{Z}) d \mathrm{Z}-\int_{0}^{\mathrm{H}} \mathrm{U}(\mathrm{X}, \mathrm{Y}, \mathrm{Z}) d \mathrm{Z}\right]$

mais : $\quad \int_{0}^{\mathrm{H}+d \mathrm{H}} U(\mathrm{X}+d \mathrm{X}, \mathrm{Y}, \mathrm{Z}) d \mathrm{Z}-\int_{0}^{\mathrm{H}} \mathrm{U}(\mathrm{X}, \mathrm{Y}, \mathrm{Z}) d \mathrm{Z}=\frac{\partial\left(\mathrm{U}_{\mathrm{I}} \mathrm{H}\right)}{\partial \mathrm{X}} d \mathrm{X}-\int_{0}^{\mathrm{H}} \mathrm{Z} \frac{\partial^{2} \mathrm{U}}{\partial \mathrm{X} \partial \mathrm{Z}} d \mathrm{X} d \mathrm{Z}$

On obtient finalement pour $(2 d)$ :

$$
\begin{aligned}
\xi_{E} W_{\mathrm{II}}(\mathrm{X}) & +\frac{\partial\left(\mathrm{U}_{\mathrm{I}} \mathrm{H}\right)}{\partial \mathrm{X}} \\
& -\int_{0}^{t} d \mathrm{Y} \int_{0}^{\mathrm{H}} Z \frac{\partial^{2} \mathrm{U}}{\partial \mathrm{X} \partial \mathrm{Z}} d Z=0
\end{aligned}
$$

Selon l'hypothèse $(c)$, v et $\xi$ sont des coefficients constants déterminés par la géométrie de la fente seulement et sont liés à $\mu$ par la relation $\xi=\nu \mu$.

En usant des variables adimensionnelles :

$$
\begin{aligned}
& x=\frac{\mathrm{X}}{\mathrm{E}_{0}} \quad u=\frac{\mathrm{U}}{\sqrt{2 \mathrm{gE}_{0}}} \quad h=\frac{\mathrm{H}}{\mathrm{E}_{0}} \\
& y=\frac{\mathrm{Y}}{t} \quad v=\frac{\mathrm{V}}{\sqrt{2 \mathrm{gE}_{0}}} \quad q=\frac{\mathrm{Q}_{0}-\mathrm{Q}(\mathrm{X})}{\mathrm{Q}_{0}} \\
& z=\frac{\mathrm{Z}}{\mathrm{E}_{0}} \quad w=\frac{\mathrm{W}}{\sqrt{2 g \mathrm{E}_{0}}}
\end{aligned}
$$

et en notant :

$$
\mathrm{F}=\int_{0}^{1} d y \int_{0}^{u} z \frac{\partial^{2} u}{\partial x \partial z} d z
$$

les équations ci-dessus deviennent :

$$
\begin{aligned}
& u_{\mathrm{I}}^{2}+w_{\mathrm{I}}^{2}=1-h \\
& u_{\mathrm{II}}^{2}+v^{2} w_{\mathrm{II}}^{2}=1 \\
& \frac{d h}{d x}=\frac{w_{\mathrm{r}}}{u_{\mathrm{I}}} \\
& \xi_{\varepsilon} w_{\mathrm{II}}+\frac{\partial}{\partial_{i}}\left(u_{\mathrm{I}} h\right)-\mathrm{F}=0
\end{aligned}
$$

On a obtenu un système différentiel du premier ordre à cinq fonctions : $\left(u_{\mathrm{I}}, w_{\mathrm{I}}, u_{\mathrm{II}}, w_{\mathrm{II}}, h\right)$ inconnues.

\section{Une solution approximative du système (3).}

On suppose que la projection horizontale de la vitesse est constante dans chaque section, c'est-à-dire :

$$
u=u(x) \text { et } u_{\mathrm{I}}=u_{\mathrm{II}}=u, \frac{\partial u}{\partial z}=0, \quad \mathrm{~F} \equiv 0
$$

Le système (3) devient :

$$
\begin{gathered}
u^{2}(x)+w_{\mathrm{I}}^{2}(x)=1-h(x) \\
u^{2}(x)+v^{2} w_{\mathrm{II}^{2}}(x)=1 \\
\frac{d h(x)}{d x}=\frac{w_{\mathrm{I}}(x)}{u_{\mathrm{I}}(x)} \\
\xi_{\varepsilon} w_{\mathrm{II}}(x)+\frac{d}{d x}[h(x) u(x)]=0
\end{gathered}
$$

et en éliminant $w_{\mathrm{I}}, w_{I \mathrm{I}}$ et $u$ on obtient

$$
\mu \varepsilon \sqrt{\frac{(d h / d x)^{2}+h}{1+(d h / d x)^{2}}}-\frac{d}{d x}\left[h \sqrt{\frac{1-h}{1+(d h / d x)^{2}}}=0\right.
$$

équation du deuxième ordre, non linéaire.

L'allure de la surface libre résulte de:

$$
\begin{aligned}
& \frac{d^{2} h}{d x^{2}}=\frac{u(d h / d x)(2-3 h)-2 \mu \varepsilon(1-h) \sqrt{1-u^{2}}}{2 h u^{2} \sqrt{1-h-u^{2}}} \\
& =\frac{(d h / d x)(2-3 h)-2 \mu \varepsilon \sqrt{1-h} \sqrt{h+(d h / d x)^{2}}}{2 u^{2} h \sqrt{1-h-u^{2}\left[1+(d h / d x)^{2}\right] 1-h}}
\end{aligned}
$$

Le signe de $d^{2} h / d x^{2}$ dépend de celui du numérateur, celui du dénominateur étant positif.

Dans l'hypothèse où $(d h / d x)>0$ (lame descendante), à l'extrémité aval de la nappe $(h \rightarrow 0$, $u \rightarrow 1)\left(d^{2} h / d x^{2}\right)$ sera positif, donc la lame sera concave.

L'allure générale du profil de la nappe dépend du signe de la seconde dérivée à l'origine, conformément à la figure 3 .

Le cas où $h_{1}<(2 / 3)$ (régime torrentiel à l'amont) est le plus intéressant (cas $c$ et $d$ ).

$$
\begin{aligned}
& h_{1}>\frac{2}{3} \begin{array}{l}
\text { régime lent } \\
\text { dans l'origine }
\end{array} \\
& h_{1}=\frac{2}{3} \text { régime critique }
\end{aligned}
$$

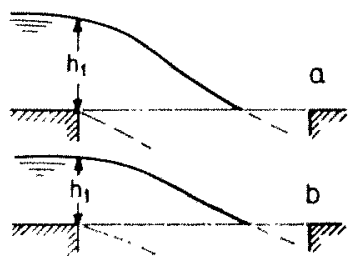

$h_{1}<\frac{2}{3}$ régime rapide

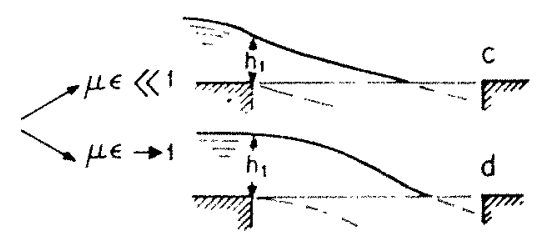

Frg. 3 
Si on admet que la pente de la nappe est petite $\left[(d h / d x)^{2}<<1\right]$, l'équation (5) devient :

$$
\text { us } \sqrt{h}-\frac{d}{d x}(h \sqrt{1-h})=0
$$

c'est-à-dire l'équation bien connue de GarotBouvard pour une grille horizontale.

Les considérations de ce chapitre montrent toutes les hypothèses que l'équation $\left(5^{\prime}\right)$ comporte et son caractère simplifié.

La prise en considération de la première dérivée $d h / d x$, nous a aussi permis d'expliquer l'apparition des nappes convexes dans le cas où les barreaux sont rares ( $\mu \in \rightarrow 1$; cas $d$ de la fig. 3 ).

\section{Le dimensionnement hydraulique des grilles ( par-dessous $)$.}

Dans les hypothèses du paragraphe 3 , on obtient toujours une correspondance qualitative avec les expériences. Il est évident qu'en réalité (comme conséquence de la courbure de la nappe) $u_{1} \neq u_{\mathrm{II}}$.

Une meilleure correspondance quantitative peut être trouvée si on ajoute une cinquième équation au système (3).

On trouvera une telle relation à l'aide des expériences. On va user du matériel publié par M. Noseda (pour les détails, voir [12], [13]).

Les données expérimentales se réfèrent à deux éléments qui peuvent être déterminés indépendamment :

A) La distribution du débit au long de la grille $Q(X)=Q_{0}-Q_{\text {derire }}$;

B) La hauteur de la nappe $\mathrm{H}=\mathrm{H}(\mathrm{X})$.

On va associer toujours une de ces relations (celle du débit A) au système (3) et on trouvera ainsi la forme de la nappe. Ce résultat sera confronté avec les mesures $B$.

a) LA DistrREUTION DU DÉBIT $Q=Q(x)$ :

Par l'élimination de $W_{\text {II }}$ des équations :

$$
\left\{\begin{array}{l}
\xi b \mathrm{~W}_{\mathrm{II}} d \mathrm{X}=d \mathrm{Q} \\
\frac{{ }^{2} \mathrm{~W}_{\mathrm{II}}^{2}}{2 g}+\frac{\mathrm{U}_{\mathrm{II}}^{2}}{2 g}=\mathrm{E}_{0}
\end{array}\right.
$$

on $\mathbf{a}$ :

$$
\frac{1}{\mu^{2} \varepsilon^{2}} \frac{\mathrm{Q}_{0}^{2}}{2 g \mathrm{E}_{0}^{3}}\left(\frac{d q}{d x}\right)^{2}+u_{1 \mathrm{I}^{2}}=1
$$

Si en amont il y a un canal :

$$
\frac{\mathrm{Q}_{0}^{2}}{2 g \mathrm{E}_{0}^{3}}=h_{0}^{2}\left(1-h_{0}\right)
$$

(pour un barrage $\left.\frac{\mathrm{Q}_{0}^{2}}{2 g \mathrm{E}_{0}{ }^{3}}=m^{2}\right)$ et on a :

$$
\frac{1}{u^{2} \varepsilon^{2}} h_{0}^{2}\left(1-h_{0}\right)\left(\frac{d q}{d x}\right)^{2}=1-u_{I I}^{2}
$$

avec la notation $\psi=\mu \varepsilon x$ :

$$
\frac{h_{0} \sqrt{1-h_{0}}}{\sqrt{h_{1}}} q=\int_{0}^{\psi} \sqrt{\frac{1-u_{\mathrm{r}}^{2}}{h_{1}}} d \psi
$$

Notons que le rapport $\sqrt{\left(1-u^{2}\right)} / h_{1}$ varie dans la section initiale de la grille 1-1 entre les limites 0 et 1 . On suppose que le long de la grille, l'intégrale de (7) sera toujours fonction de $\psi$.

En représentant les données expérimentales [12], [13] dans un graphique où

$$
\left[h_{0} \sqrt{\left(1-h_{0}\right)} / \sqrt{h_{1}}\right] q
$$

est reporté comme fonction de $\varepsilon x$, on obtient une courbe unique, résultant d'expériences effectuées dans des conditions diverses (fig. 4), ce qui confirme le caractère général de la relation (7).

\section{b) LA FORME DE LA NAPPE :}

Le système différentiel (2) peut être réduit à l'équation :

$$
\begin{aligned}
& h_{0} \sqrt{1-h_{0}} q \\
& =\int_{0}^{x}\left[\frac{d}{d x}\left(h \sqrt{\frac{1-h}{1+(d h / d x)^{2}}}\right)-\mathrm{F}\right] d x
\end{aligned}
$$

La limite inférieure de l'intégrale peut être prise en amont, dans la zone à pression hydrostatique, au lieu de la section 1-1, parce que $q=0$ en amont de la grille.

On obtient ainsi :

$$
\begin{aligned}
& \int_{0}^{x}\left[\frac{d}{d x}\left(h \sqrt{\left.\frac{1-h}{1+(d h / d x)^{2}}\right)-\mathrm{F}}\right] d x\right. \\
& =h_{0} \sqrt{1-h_{0}}-\left[\frac{h \sqrt{1-h}}{\sqrt{1+(d h / d x)^{2}}}-\int \mathrm{F} d x\right]
\end{aligned}
$$

L'intégrale :

$$
\int F d x=\int_{0}^{x} d x \int_{0}^{1} d y \int_{0}^{h} z \frac{\partial^{2} u}{\partial z \partial x} d z
$$

peut être négligée parce que la non uniformité des vitesses (de laquelle $\partial^{2} u / \partial x \partial z$ dépend) est concentrée à l'entrée de la grille et au fond. 


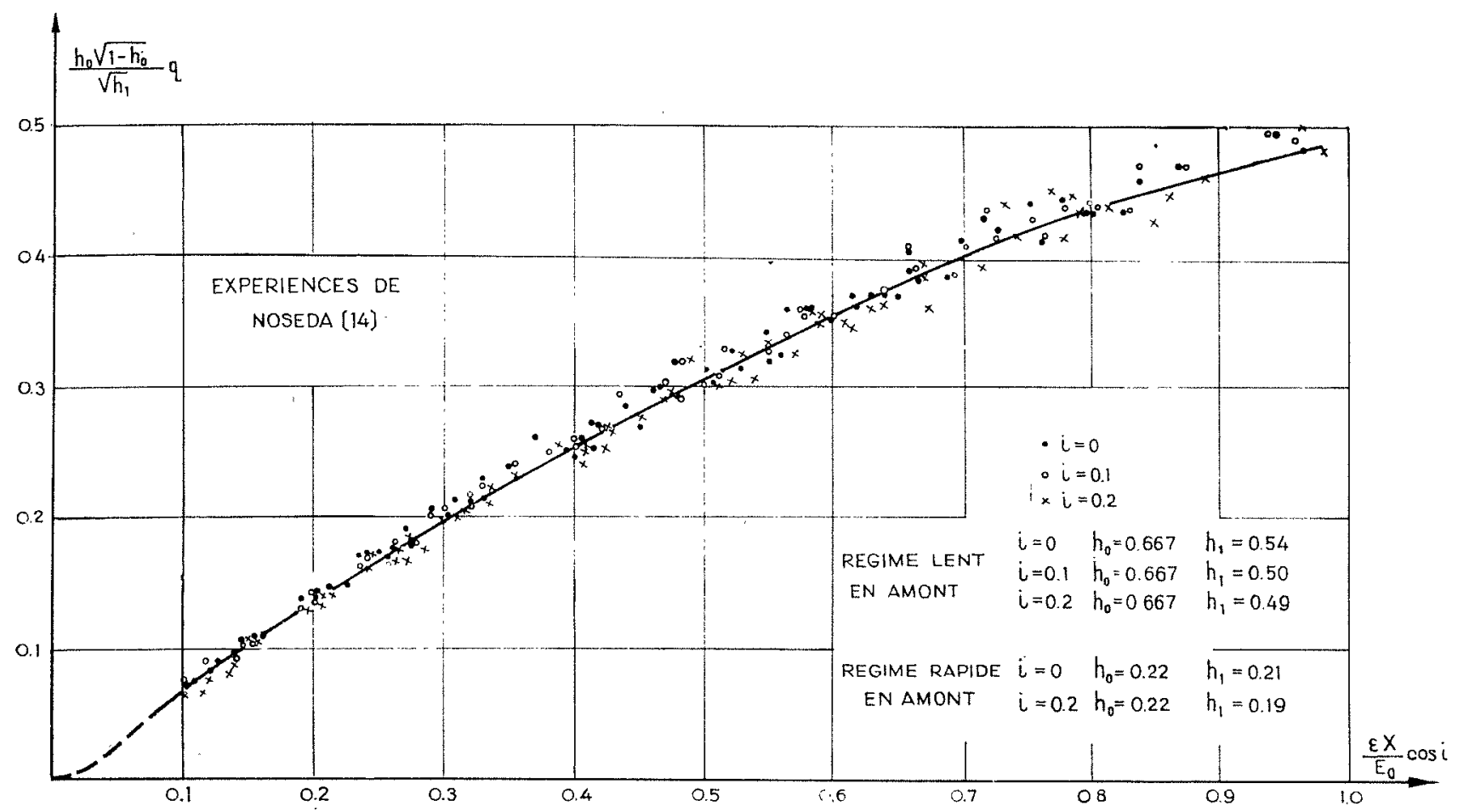

Fig. 4

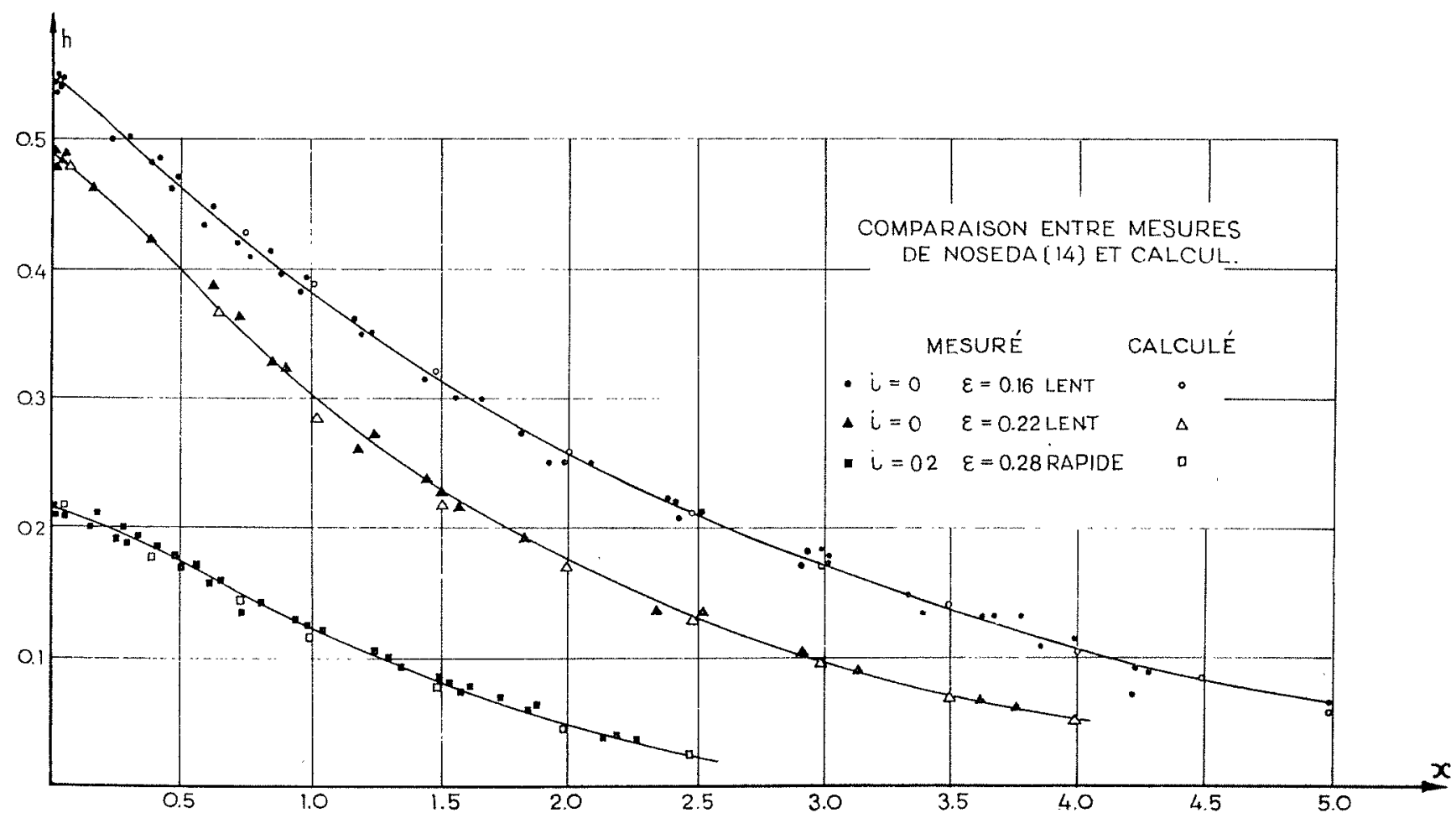

FIG. 5 
On arrive ainsi aux relations :

$$
h \sqrt{\frac{1-h}{1+(d h / d x)^{2}}}=h_{0} \sqrt{1-h_{0}}(1-q)
$$

pour les grilles emplacées au fond des canaux et :

$$
h \sqrt{\frac{1-h}{1-(d h / d x)^{2}}}=m(1-q)
$$

pour les grilles emplacées sur la crête d'un barrage.

Pour les grilles moins rares (expériences de Noseda) $(d h / d x)^{2}<<1$ et l'expression (8') est plus simple encore :

$$
h \sqrt{1--h}=h_{0} \sqrt{1-h_{0}}(1-q)
$$

Il est admis de négliger $(d h / d x)^{2}$, sauf dans le cas où $h_{1}=(2 / 3)$ uniquement, puisque, au voisinage de cette valeur, $h$ varie fortement, même pour de petites variations de :

$$
f(h)=h \sqrt{1-h} \quad\left(\frac{d h}{d f}=\frac{1}{3 h[(2 / 3)-h]}\right)
$$

Les résultats obtenus à l'aide de (9) et (7) concordent très bien avee les expériences de M. Noseda. Dans la figure 5, on donne deux exemples de comparaison entre les profils calculés et mesurés.

On a obtenu encore une bonne concordance avec l'expérience décrite dans [16] aussi, mais cette fois $d h / d x$ n'a plus été négligée (formule $8^{\prime \prime}$ ) et le profil a été déduit par les différences finies (fig. 6).

\section{c) INFLUENCE DE la PENTE dE LA GRILIE:}

En admettant les mêmes hypothèses (qui sont valables pour les pentes usuelles $(i \leq 0,2))$, il est possible d'écrire de nouveau le système fondamental (avec les notations de la fig. 7).

$$
\begin{aligned}
& u_{\mathrm{I}}^{2}+w_{\mathrm{I}}^{2}=1+x \sin i-h \operatorname{séc} i \\
& u_{\mathrm{II}}^{2}+v^{2} w_{\mathrm{II}}^{2}=1+x \sin i \\
& \frac{d h}{d x}=\frac{w_{\mathrm{I}}}{u_{\mathrm{I}}} \\
& \check{\zeta}_{\varepsilon} w_{\mathrm{II}}+\frac{d}{d x}\left(u_{\mathrm{I}} h\right)-\mathrm{F}=0
\end{aligned}
$$

La relation empirique entre $q$ et $x$ recevra la forme

$$
\frac{h_{0} \sqrt{1-h_{0}}}{\sqrt{h_{1}}} q=f(\downarrow) \quad \psi=\mu . \varepsilon \cos i x
$$

Sur la figure 4 on a représenté les points correspondant aux expériences de $M$. Noseda ( $i=0,1$ et $i=0,2$ ). On constate une bonne coïn- cidence avec le cas $i=0$ con a excepté les expériences à $i=0,2$, régime rapide en amont, $\mathrm{Q}_{0}=155,4 \mathrm{l} / \mathrm{s} . \mathrm{m}$ et $\mathrm{Q}_{0}=198,3 \mathrm{l} / \mathrm{s} . \mathrm{m}$, où les pertes de charge sont probablement sensibles).

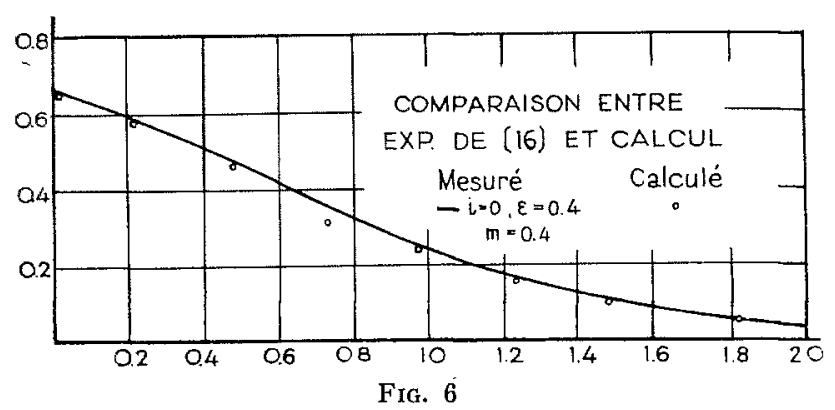

La fonction $f(\psi)$ (fig. 8), valable au moins entre les limites expérimentales de M. Noseda, $[\varepsilon=0,16 \div 0,40 ; q \leqslant 0,9 ; i=0 \div 0,2 ; \mu=0,72]$ a donc un caractère très général, puisqu'elle contient dans une relation unique les influences de la forme et de l'épaisseur des barreaux, du régime en amont, de la pente et du débit.

d) Example de calcul de la longueur de la GRILLE :

$1^{\circ}$ Choisir la longueur d'une grille «par-dessous $\gg$ caractérisée par :

- canal en amont;

- régime lent en amont;

- pente de la grille $i=0,1$;

- barreaux à arêtes vives;

$-s=(b / t)=0,20$;

$-\mathrm{Q}=0,5 \mathrm{~m}^{3} / \mathrm{s} . \mathrm{m}$;

- la grille doit capter $80 \%$ du débit maximal.

Pour calculer la longueur, il faut déterminer les paramètres $h_{1}, h_{0}$, ц., $q, \mathrm{E}_{0}$ :

- d'après Noseda [12] [13] $h_{1}=0,50$;

- supposons qu'en amont le régime soit critique $h_{0}=0,67$;

- pour une fente à mince paroi, d'après la courbe de Mises [15] $\mu_{0}=0,615$;

$$
\begin{aligned}
-q & =\frac{\mathrm{Q}_{0}-\mathrm{Q}}{\mathrm{Q}_{0}}=0,8 ; \\
-\mathrm{E}_{0} & =\sqrt[3]{\frac{\mathrm{Q}_{0}^{2}}{2 g h_{0}^{2}\left(1-h_{0}\right)}} \\
& =\sqrt[3]{\frac{0,5^{2}}{2 \times 9,81 \times 0,667^{2} \times 0,33}}=0,44 \mathrm{~m} .
\end{aligned}
$$

On a done :

$$
\frac{h_{0} \sqrt{1-h_{0}}}{\sqrt{h_{1}}} q=\frac{0,667 \times \sqrt{0,33}}{\sqrt{0,50}} \times 0,8=0,435
$$


Selon le graphique de la figure 8 , à cette valeur correspond :

$$
\psi=\frac{\mu \cdot \mathrm{X} \cos i}{\mathrm{E}_{0}}=0,56
$$

La longueur de la grille sera :

$$
\begin{aligned}
\mathrm{L}=\mathrm{X}=0,56 & \frac{\mathrm{E}_{0}}{\mu_{\mathrm{\varepsilon}} \cos i} \\
& =\frac{0,56 \times 0,44}{0,615 \times 0,25 \times 0,951} \cong 1,70 \mathrm{~m}
\end{aligned}
$$

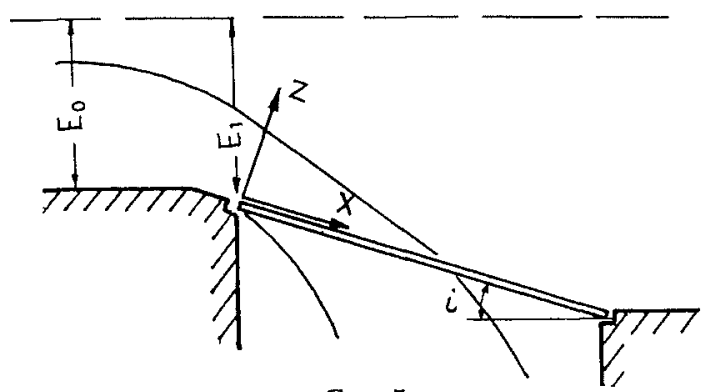

FIG. 7

$2^{\circ}$ Déterminer Ia Iongueur d'une grille pardessous :

- emplacée sur la crête d'un barrage à coefficient de débit $m=0,40$;

- pente $i=0,15$;

- barreaux à arêtes arrondies;

$-\varepsilon=0,35$;

- $\mathrm{Q}_{0}=0,5 \mathrm{~m}^{3} / \mathrm{s} . \mathrm{m}$;

- la grille doit capter $75 \%$ du débit.

D'après Mostcoff [17] $h_{1}=0,449$;
D’après Noseda [12] $\mu=0,72$ :

$$
\begin{gathered}
\mathrm{E}_{0}=\sqrt[3]{\frac{\mathrm{Q}_{0}^{2}}{2 g m^{2}}}=\sqrt[3]{\frac{0,5^{2}}{2 \times 9,81 \times 0,40^{2}}}=0,43 \mathrm{~m} \\
\frac{m q}{\sqrt{h_{1}}}=\frac{0,40 \times 0,75}{\sqrt{0,449}}=0,447
\end{gathered}
$$

selon la figure 8 :

$$
\begin{gathered}
\psi=\frac{\mu \varepsilon \mathrm{X} \cos i}{\mathrm{E}_{0}}=0,60 \\
\mathrm{~L}=\mathrm{X}=\frac{0,43 \times 0,60}{0,72 \times 0,35 \times 0,888}=1,16 \mathrm{~m}
\end{gathered}
$$

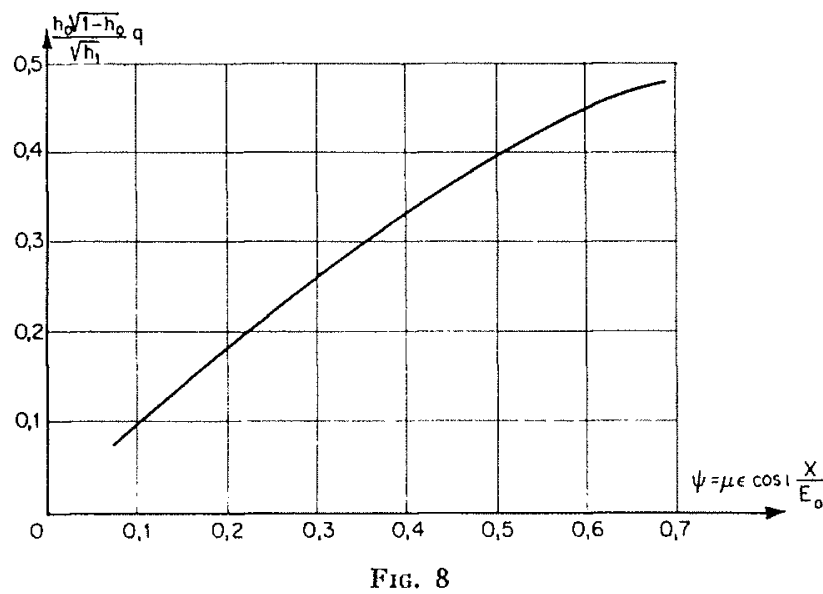

Le calcul hydraulique de la grille fournit toujours les données de base; dans de nombreux cas, il faut tenir compte de l'obstruction de la grille.

\section{BIBLIOGRAPHIE}

[1] Bovvard (M.). - Le débit d'une grille «par-dessous 》. - La Houille Blanche, $\mathrm{n}^{\circ} 2$ (1953).

[2] Daniela (H. F.) et Bobohidze (S. S.). - Prise d'eau à grille. - Ghidrotehnitscheskoe Stroitel'stvo, $\mathrm{n}^{\circ} 11$ (1956), Moscou, U.R.S.S.

[3] Frank (I.). - - Recherche hydraulique sur les prises tyroliennes. - Der Bauingenieur, $\mathrm{n}^{\circ} 31$ (1956).

[4] Fandeev (V.V.). - Déficiences des barrages placés sur les cours d'eau des régions montagneuses. Ghidrotehnica $i$ Melioratzia, $\mathrm{n}^{\circ} 3$ (1956), Moscou, U.R.S.S.

[5] Fandeev (V. V) et MrrZa-Zade (V. R) - La capacité de dérivation des barrages à grilles «par-dessous». - Ghidrotehnica i Melioratzia, $n^{\circ} 3$ (1952), Moscou U.R.S.S.

[6] Kuntzman (J.) et Bouvard (M.). - Etude théorique des grilles des prises d'eau «par-dessous». - La Houille Blanche, $\mathrm{n}^{\circ} 5$ (1954).

[7] Mexic-Nubarov (C.). - Le calcul hydraulique d'une grille "par-dessous 》. - Ghidrotehnitschescoe Stroitel'stvo, $\mathrm{n}^{\circ} 11$ (1956), Moscou, U.R.S.S.

[8] Zamarin et FandeEv. - Constructions hydrotechniques. - Moscou, 1952.
[9] Fandeev (V. V.). - Sur le calcul des barrages à grilles «par-dessous». - Ghidrotehnica $i$ Melioratzia, $\mathrm{n}^{\circ} 7$ (1953), Moscou, U.R.S.S.

[10] Melic-Nubarov (C.). - Calcul et exploitation des prises «par-dessous». - Ghidrotehnitschescoe Stroitel'stvo, $\mathrm{n}^{\circ} 3$ (1952), Moscou, U.R.S.S

[11] Mostcoff (M. A.). - Aide-mémoire hydraulique.

[12] Noseda $(G$.$) . - Correnti permanenti con portata$ progressivamento decrescente, defluenti su griglie di fondo. Ricerca sperimentale. - L'Energia Elettrica, $\mathrm{n}^{\circ} 6$ (1956), pi 565.

[13] Noseda (G.) - Sul calcolo idraulico delle griglie di fondo. - L'Energia Elettrica. $\mathrm{n}^{\circ} 12$ (1956), p. 1347.

[14] Onth (J.), Chardonnet (E.) et Meynardi (G.). L'étude des grilles pour prises d'eau du type «pardessous $\gg$. - La Houille Blanche, $\mathrm{n}^{\circ} 3$ (1954).

[15] Rouse (H.). - Engineering Hydraulics, Iowa, 1949.

[16] Wisner (P.), Triteanu (0.), Mrtric (A.) et Drimmer (G.). - Etude sur modèle et dans la nature des captations secondaires. - Hidrotehnica, $\mathrm{n}^{\circ} \quad 6$ (1957), Bucarest, Roumanie.

[17] Mostcoff (M. A.). -- Sur le calcul des grilles des prises d'eau. - La Houille Blanche, $\mathrm{n}^{\circ} 4$ (1957). 